\title{
OBERE UND UNTERE SCHRANKE FÜR DIE KOMPLEXITÄT VON BOOLESCHEN FUNKTIONEN
}

\author{
Banke Bremer \\ Institut fir angewandte Mathematik \\ Joh. Wolfg. Goethe Univ. Frankfurt
}

Eine Menge Boolescher Funktionen heiBe n-stellig, wenn alle enthaltenen Funktionen auf ỏen gleichen $n$ Variablen definiert sind. Alle n-stelligen Mengen der Mächtigkeit $t$ werden zu

$$
M_{t, n}=\{F|| F \mid=t, F \text { ist } n-s t e l i i g\}
$$

zusammengefaßt. Im folgenden soll eine obere und eine untere Schranke fir die Komplexität von $F$ in Abhängigkeit von $n$ und $t$ angegeben werden. Zur Berechnung von $F$ seien als o-stellige Operationen mit den Kosten o die Konstanten $o$ und 1 und die Projektionen $x_{1}, \ldots, x_{n}$ zugelassen, als 2-stelifge Operationen die Multiplikation und die Addition mod 2 mit den Kosten 1.

\section{Lemma 1}

Fiir die Komplexität $L(F)$ einer Menge Boolescher Funktionen F aus $M_{t, n}$ gilt

$$
I(F) \leq \frac{t 2^{n}}{l d t}\left(1+c \frac{l d I d t}{l d t}\right)
$$

Darin ist $c$ eine von $n$ und $t$ unabhängige Konstante. 
Der Beweis ist in [1] angegeben, jedoch obne Abschätzung des Fehlergliedes. Dazu werden erst alle Produkte aus Variablen und negierten Variablen berechnet. Diese $2^{n}$ Produkte werdea in $\left\lceil 2^{n} / p\right\rceil$ Gruppen zu je $\mathrm{p}$ Stück geteilt. In jeder Gruppe werden alle Linearkombinationen berechnet, aus denen dann durch Addition die $t$ Ergebnisse zusamnengesetzt werden. Es kommt die Schranke des Lemmas heraus, die jedoch nur fur grobe t gut ist. Deshalb der folgende Satz.

\section{Satz 2}

Für die Komplexitat $\mathrm{I}(\mathrm{F})$ einer Menge Boolescher Funktionen $F$ aus $\mathrm{M}_{t, n}$ gilt

$$
L(F) \leq \frac{t 2^{n}}{1 a t 2^{n}}\left(1+c \frac{1 d I d t 2^{n}}{1 a t 2^{n}}\right)
$$

Darin ist $c$ eine von $\mathrm{n}$ und $t$ unabhangige Konstante.

Der Beweis ist für eine Funktion ohne Abschätzung des Feblergliedes in [1] gegeben. Es muß jedoch der Parameter m genauer gewählt und schärfer abgeschätzt werden, damit das Verfahren für Mengen F kleiner Machtigkeit zum Ziel führt. Fü große $F$ reicht schon Lemma 1 aus. Eine Berechnung von $\left.\mathbb{F}=C_{f_{1}}, \ldots, f_{t}\right\} \in M_{t, n}$ ist gegeben durch die Zerlegung

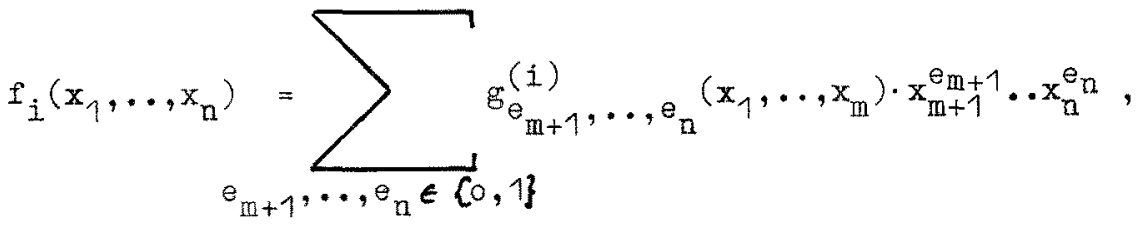

worin die Funktionenmenge $G=\{g(i)(\ldots)\}$ in $M_{s, m}$ mit $s \leq t 2^{n-m}$ Iiegt. $G$ wird nach dem Lemma 1 berechnet und $F$ aus $G$ und den restlichen Variablen gemä der Zerlegung.

Nun soll eine untere schranke für L(F) von der gleichen Größenordaung gezeigt werden, wozu jedoch zwei Ausnahmen zu machen sind. Einmal mussen allzu einfache Funktionen ausgenommen werden, die aber nur selten auftreten. Außerdem führt die angewendete Abzählung der Berechnungen nur für relativ kleine Mengen $F$ zum Ziel. Deshalb die zwei folgenden Definitionen. 


$$
M=\underbrace{M_{t, i n}}_{t \leq 2^{2}}
$$

NCM heißt Nullmenge, wenn

$$
\lim _{t 2 \rightarrow \infty} \frac{\left|M_{t, n} \cap N\right|}{\left|M_{t, n}\right|}=0 .
$$

\section{Satz 3}

Für fast alle $F$ aus $M$, d. h. für alle außerhalb einer Nullmenge $N$, gilt

$$
I(F) \geq \frac{t 2^{n}}{1 d t 2^{n}}\left(1-\frac{1 a l a t 2^{n}}{1 d t 2^{n}}\right),
$$

falls $F$ aus $M_{t, n}$.

Zum Beweis des Satzes werden alle $F$ aus $M$, für die die Ungleichung aicht gilt zur Menge $N$ zusammengefaßt. Ts bleibt zu zeigen, daß $N$ eine Nullmenge ist, wozu die normalen Berechnungen mit vorgegebener Länge wie in 61 abgezăhlt werden.

Nun ist eine obere und eine untere Schranke für die Komplexität von Mengen Boolescher Funktionen angegeben. Weiterhin ist die Lücke zwischen diesen beiden Schranken klein und bekannt, so daß es nun möglich ist, Ersparnisse bei gemeinsamer Berechnung anzugeben. Definiert mar otwa die Ersparnis durch

$$
E=\frac{L\left(f_{1}\right)+\ldots+I\left(f_{t}\right)-I\left(f_{1}, \ldots, f_{t}\right)}{t 2^{n} / n}
$$

so kommt

$$
E \gtrless \frac{1 d t}{n+1 d t} \mp 2 c \frac{1 d n}{n}
$$


heraus. Genaue Angaben über die Ersparnis sind also nur möglich, wenn ldt $\gg$ ldn ist. So erhält man etwa bei gemeinsamer Berechnung von $2^{n}$ Funktionen, die nicht in der Nullmenge liegen, für große n eine Erspamis von genau 50\%. Selbst bei Berechnung von relativ wenig Funktionen im Vergleich zu allen kan man also erheblich sparen.

[1] V. STRASSEN:

Berechnungen in partiellen Algebren endiichen Typs Computing $11 / 3$

[2] D. E. MULLER:

Complexity in Electronic Switching Circuits

IRE Transactions on Electronic Computers, March 1956

[3] H. BREMER:

Berechnungskomplexitat von Mengen Boolescher Funktionea und von Polynomen uber endilchen Körpern Diplomarbeit, Institut für angewandte Mathematik an der Johann Wolfgang Goethe Universität in Frankfurt 